\title{
Intervencionismo en oclusiones crónicas: evaluación de la efectividad cruce de distintas guías y balones según complejidad.
}

Pablo Pedreros, Rubén Lamich, Arnoldo Aguirre, Carlos Romero, Hernán Chamorro.

Departamento de Hemodinamia, Hospital Barros Luco Trudeau.

\section{Resumen:}

Antecedentes: El intervencionismo en las oclusiones totales crónicas (OTC) requiere técnicas complejas y el éxito es poco predecible. Es útil identificar la efectividad de las guías de cruce y del balón para el intervencionismo en distintos niveles de complejidad de OTC.

Objetivos: Evaluar la efectividad de cruce de distintas guías y balones en el tratamiento de OTC.

Métodos: Se revisa en forma retrospectiva la efectividad de distintos tipos de guías y balones de cruce utilizados en intervencionismo de OTC efectuados entre agosto de 2007 y agosto de 2009. Se define "efectividad" como la capacidad de avanzar y cruzar la oclusión crónica con la guía y el balón, ambas necesarias para efectuar la angioplastia de la OTC. Se agrupan los resultados según el nivel de complejidad de la OTC de acuerdo a score propio, distinguiendo entre OTC simples, complejas y de alta complejidad.
Resultados: En el período estudiado se revisan 90 OTC. Se utilizan 148 guías (1,6 guía/proc.) y se utilizan 92 balones en 76 procedimiento que logran paso efectivo de guía. ( $1,2 \mathrm{bal} / \mathrm{proc}$.). Se realiza una tabla con la tasa de efectividad de distintas guías y balones de cruce. Destaca la utilidad de guía PT2 en lesiones simples y complejas, como la utilidad de la guía Miracle 6 en OTC de alta complejidad.

Conclusiones: En OTC se obtiene un éxito de $67 \%$ en nuestro medio, con mayor efectividad de la guía PT2 en lesiones simples y complejas y de Miracle 6 en lesiones de alta complejidad. En cuanto a balones se obtiene mayor efectividad en los tipo Maverick, Mercury y Voyaguer de $1,5 \mathrm{~mm}$ en lesiones simples y complejas, como del Balón Maverick de 1,5 mm en lesiones de alta complejidad. Es de gran utilidad un registro de la efectividad de balones y guías en el tratamiento de OTC, lo que permite al intervencionista una adecuada elección del material con el consecuente ahorro de tiempo y mayor efectividad del procedimiento.

Correspondencia:

Dr. Pablo Pedreros Bettini.

Mail: ppbettini@yahoo.com 


\section{Percutaneous coronary interventions in chronic coronary occlusions: effectiveness of different guides and balloons according to complexity of total coronary artery occlusion}

Background. : PCI in chronic coronary occlusions requires complex techniques and is associated to less predictable results. It is important to evaluate the effectiveness of different guides and balloons in $\mathrm{PCI}$ for total coronary occlusion (TCO).

Aim: To evaluate the effectiveness of different guides and balloons in the treatment of TCO.

Methods: A retrospective review of the effectiveness of different guides and balloons in the treatment of TCO performed from 2007 to 2009 was carried out. Effectiveness was defined as the ability to traverse the occlusion with the guide and the balloon. The analysis was performed according to groups defined by the complexity of the TCO as assessed by a locally developed score. Occlusions were classified as simple, complex or highly complex.

Results: 90 procedures were analyzed. 148 guides were utilized (mean of 1.6 per procedure) and 92 balloons were

\section{Introducción:}

Se define oclusión total crónica (OTC) como aquella obstrucción coronaria completa (flujo TIMI 0 en la zona ocluida), que tiene al menos 3 meses de evolución ${ }^{1}$. Por el tiempo de evolución, generalmente la zona ocluida esta ricamente fibrótica y a veces calcificada. La apertura vía percutánea del vaso en una OTC es, en general, un procedimiento de mayor dificultad. $\mathrm{El}$ intervencionismo en OTC se considera un procedimiento complejo que requiere mayor destreza, utilización de materiales sofisticados, mayor cantidad de tiempo empleado y alta probabilidad de complicaciones. Además, el resultado es incierto, con una tasa de éxito de 50 a $80 \%$ dependiendo de la experiencia del Centro.

A nuestro juicio el éxito en el intervencionismo de oclusiones crónicas depende de las técnicas aplicadas, pero en un gran porcentaje este éxito puede ser determinado por el tipo de material utilizado. Por otra parte, mientras más complejo y agresivo sea el material utilizado, este se puede asociar a una mayor tasa de complicaciones.

Es importante elegir el material adecuado según la used in 76 cases were the guide successfully crossed the occlusion. The PT2 guide was effective for simple and complex lesions, whereas de Miracle 6 guide was effective in the treatment of highly complex lesions. Regarding balloons, the Maverick, Mercury and Voyageur $1.5 \mathrm{~mm}$ were satisfactory in simple and complex lesions, while the Maverick $1.5 \mathrm{~mm}$ was more effective in highly complex lesions

Conclusion: TCO was successfully treated by PCI in $67 \%$ of cases. PT2 guides and all types of balloons were effective for treatment of simple and complex lesions. In contrast, the Miracle 6 guide and the Maverick 1.5 balloon were more effective in highly complex lesions. These findings may be useful to help de proper selection of angioplasty materials when treating TOC.

Key words: Total chronic occlusions, PTCA.

habilidad del operador y la severidad de la OTC para lograr mejores resultados y evitar una alta tasa de complicaciones.

No existen actualmente publicaciones sobre la efectividad de las distintas guías y balones en el tratamiento de las OTC, por lo que los operadores basan su elección en las características netas de los materiales y en la experiencia propia. Se ha publicado experiencias de utilización de dos tipos de guías como estrategias comparativas, lo que da ideas limitadas en cuanto a las posibilidades de elección del material ${ }^{2}$.

Pensamos que para aquellos grupos que se inician en estas técnicas sería de utilidad el saber qué material utilizar, con qué tipo de lesión y cuándo el material utilizado puede asociarse a mayor tasa de éxito.

El propósito de este estudio es crear una guía, producto de nuestra experiencia en el tratamiento de las OTC, que muestre la efectividad en el paso de las oclusiones por parte de distintos tipos de guías y de balones de angioplastía. Para una mejor selección del material hemos clasificado las oclusiones crónicas de acuerdo al nivel de complejidad según un score propio de OTC elaborado en nuestro laboratorio. Basado en este Score ajustamos la tasa de efectividad de las guías y balones 
Gráfico 1: Riesgo relativo de éxito (con IC), calculado para cada factor presumiblemente Influyente en el resultado de la angioplastía.

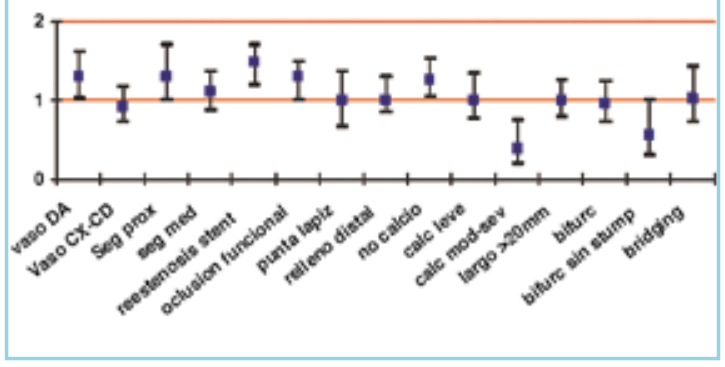

según el nivel de complejidad de la OTC.

\section{Métodos:}

Se revisa en forma retrospectiva todas las angioplastías de OTC efectuadas en el laboratorio de Hemodinamia del Hospital Barros Luco, en el período comprendido entre agosto de 2007 y agosto de 2009. Se estudia un total 90 angioplastías. Por cada angioplastía se realiza un detallado registro del material utilizado en cuanto al número y tipos de guías, como al número y tipos de balón. Por cada tipo de guía y balón evaluamos su efectividad.

\section{Score de complejidad de OTC:}

Clasificamos las oclusiones como simples, complejas o de alta complejidad, de acuerdo al score de complejidad elaborado en nuestro laboratorio de la siguiente forma:

Se identifican 15 parámetros angiográficos que pueden influir en el éxito o fracaso de la angioplastía sobre la OTC. No se utiliza como parámetro el tiempo de oclusión ya que este dato muchas veces no se puede precisar. De los datos analizados, según el comportamiento en los 90 casos realizados, se define el riesgo relativo (RR) de éxito, con su intervalo de confianza (IC), (gráfico 1). Aquellos parámetros que son predictivos (estadísticamente significativos) en el éxito o fracaso del procedimiento, son considerados para el crear el score.

Para este score se consideran los siguientes parámetros angiográficos: 1) Vaso afectado, 2) segmento del vaso afectado, 3) severidad de la calcificación en la zona ocluida (moderada o severa), 4) presencia de bifurcación sin stump, 5) caracterización funcional de la oclusión, o 6) oclusión total de stent implantado previamente. Todas estas características son individualmente predictivas de éxito o fracaso en el tratamiento de la OTC. Se asigna puntaje a cada característica con un score de 1 a 8 (tabla 2).

Luego se clasifican las OTC en simples (puntaje 1 a 4), complejas (puntaje de 5 a 6 ) o de alta complejidad (puntaje de 7 a 8) (tabla 3). Este score nuestro es predictivo en cuanto a éxito del procedimiento y en cuanto al tiempo y contraste empleado ${ }^{3}$ (gráficos $2,3,4$ ).

Procedimiento de angioplastía sobre OTC:

Aunque el procedimiento de angioplastía en este grupo de pacientes es inicialmente standard, la estrategia varía significativamente según el carácter de la lesión y la experiencia adquirida, constituyendo este hecho en

\section{Tabla 2: Score de complejidad: aplicaci $\mathbf{n}$ de puntos seg $\mathbf{n}$ la presencia o ausencia de factores de riesgo de xito/fracaso.}

\begin{tabular}{|c|c|c|c|}
\hline Factor & $\begin{array}{c}\text { xito. } \\
\text { (Total: } 67 \%)\end{array}$ & P (todos sig) & Puntaje \\
\hline Vaso: DA. & $86 \%$ & 0,023 . & Ausencia: $+1 p$ \\
\hline Segmento proximal. & l. $81 \%$ & 0,06 . & Ausencia: $+1 p$ \\
\hline \multicolumn{4}{|l|}{ Calcificacion: } \\
\hline \multirow[t]{2}{*}{ Moderada o sev. } & $33 \%$ & 0,0019 . & Mod: $+2 p$ \\
\hline & & & Sev: $+3 p$ \\
\hline Restenosis stent. & $100 \%$ & 0,002 & Ausencia: $+1 p$ \\
\hline Oclusion funcional. & $86 \%$ & 0,002 . & Ausencia: $+1 p$ \\
\hline Bifur. sin stump. & $36 \%$ & 0,0029 . & $+1 p$ \\
\hline Total & & & 8 ptos \\
\hline
\end{tabular}

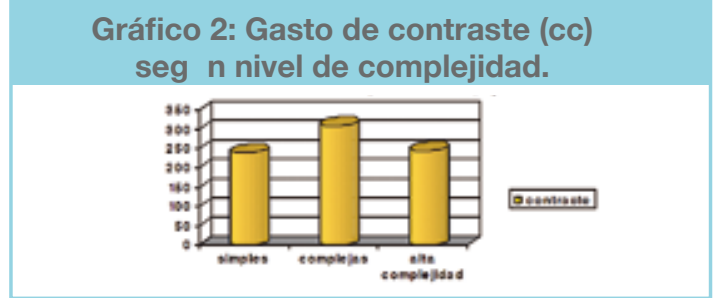

Gráfico 3: tiempo empleado (min) según nivel de complejidad.
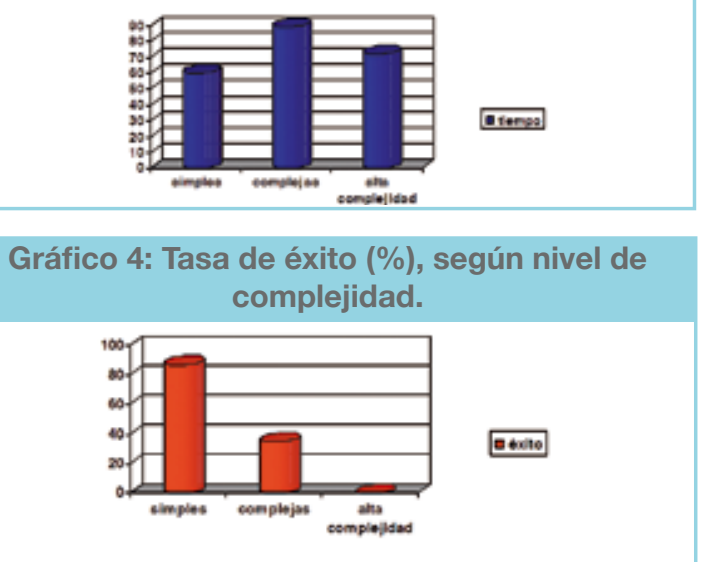
Tabla 3: Definición de complejidad de OTC según Score

Índice de complejidad Score:

1-4: simples.

5-6: complejas.

7-8: alta complejidad.

si, una limitante para el estudio.

El abordaje radial derecho es la vía de elección (52\%), femoral derecho en $22 \%$, radial izquierdo o femoral izquierdo en un $2 \%$ cada uno. Se utilizó abordaje bilateral en $22 \%$.

Los catéteres guía utilizados varían según el vaso tratado. En el $92 \%$ de los casos se ocupó 6 French. Principalmente se utilizó EBU 3,5 (16\%) en el tratamiento de OTC de descendente anterior, AL-2 (21\%) en el caso de OTC de circunfleja y AL-2 y MAC 3,5 (7\%) en el tratamiento de OTC de coronaria derecha.

Se empleó microcateter en un $23 \%$ a criterio del operador, técnica de visualización distal con inyección retrograda en un $20 \%$. Se realizó técnica perforante y drilling en un $77 \%$, mientras que técnicas de disección programadas como STAR lo fue en un $16 \%$.

En 8 pacientes se intentó vía retrograda (9\%) la que fue transeptal en 7 pacientes y tras epicárdica en 1 paciente.

La elección inicial de guía fue principalmente Galeo Floppy (16\%) (Floppy no hidrofílica) y PT2 (68\%) (Intermedia hidrofílica), apoyado en un balón monorraíl de pequeño tamaño en un procedimiento que denominamos "reconocimiento de la lesión", lo que nos muestra la dureza de la placa a tratar, la limitación de torque del vaso, problemas de angulación, etc. Muchas veces se logra traspasar la lesión con este procedimiento, en caso que la placa sea blanda o la oclusión sea funcional. De lo contrario la elección de la siguiente guía dependerá del criterio del operador apoyado en las características angiográficas y "de reconocimiento" de la lesión.

\section{Cruce de guías y balones:}

Definimos "efectividad" como la capacidad de avanzar y cruzar la oclusión crónica por parte de la guía o del balón. Ambas situaciones son necesarias para efectuar en forma exitosa la angioplastía de la OTC. El cruce se define como el paso a través del segmento del vaso ocluido (con flujo TIMI 0) y el posicionamiento de la guía o del balón en el lumen verdadero distal del vaso a tratar.

Con estos datos se realizan tablas que muestran la efectividad de los distintos tipos de guías y distintos tipos de balones en el tratamiento de las OTC. Se evalúa la efectividad total y para cada grupo de complejidad de OTC.

Los datos son expresados en número total de guías o balón utilizados vs el número total de guías o balón con cruce efectivo. La relación de ambos se expresa en porcentajes.

\section{Análisis estadístico:}

Los datos se expresan en números totales y promedios $\pm \mathrm{SD}$.

Para el diseño del score de complejidad se realizó el análisis de cada factor de riesgo con test de X2 y análisis de p con test exacto de Fisher. Para el análisis de RR e IC se utilizó el programa estadístico Statview (tablas de contingencia). Aquellos parámetros que presentan significancia estadística son empleados en la confección del score. La formación de grupos según puntaje de complejidad se realiza en forma arbitraria.

Las tasas de efectividad se expresan en porcentaje de éxito v/s total empleado.

\section{Resultados:}

Se revisan 90 angioplastías sobre OTC, lo que corresponde a un $6 \%$ del total de angioplastías efectuada en este período (1500).

Las características clínicas y angiográficas de los pacientes se presentan en la tabla 1. La edad promedio es $61+$ +- 8 años. El 70\% de los pacientes tenía diagnóstico de angina estable y en un $92 \%$ enfermedad multivaso. La arteria coronaria más frecuentemente tratada es la CD (44\%).

Se utilizan 148 guías (1,6 guía/proc.) en 90 procedimientos y 92 balones en 76 procedimiento que logran paso efectivo de guía (1,2 bal/proc.). La tasa de éxito de procedimiento fue de $67 \%$ (61 de 90). Se agrupan las angioplastías de acuerdo al grado de complejidad en simples (62p), complejas (20p) o de alta complejidad (8p) de acuerdo a nuestro propio score de complejidad. La tabla 4 muestra la tasa de efectividad para cada tipo de guía en general y agrupados de acuerdo al grado de complejidad. Destaca la utilidad de guía PT2 en lesiones simples y complejas, como la utilidad de la guía Miracle 6 en OTC de alta complejidad.

La tabla 5 muestra la tasa de efectividad para cada tipo de balón según marca y dimensiones. También se agrupan los resultados en general y de acuerdo al grado e 


\begin{tabular}{|c|c|c|}
\hline & Pacientes $\mathrm{n}=90$ & porcentaje \\
\hline edad & $61( \pm 8)$ años & \\
\hline Hombres & 69 & $76 \%$ \\
\hline Hipertensión. & 74 & $82 \%$ \\
\hline Diabetes. & 14 & $15 \%$ \\
\hline Dislipidemia. & 40 & 44 \\
\hline Tabaquismo. & 25 & $27 \%$ \\
\hline \multicolumn{3}{|l|}{ Diagnostico: } \\
\hline \multirow[t]{2}{*}{ SCA Angina estable. } & 31 & $35 \%$ \\
\hline & 59 & $65 \%$ \\
\hline Enfermedad multivaso. & 83 & $90 \%$ \\
\hline Vaso con OTC ADA & 22 & $24 \%$ \\
\hline Circunfleja. & 28 & $31 \%$ \\
\hline CD. & 40 & $44 \%$ \\
\hline
\end{tabular}

complejidad de la OTC. Destaca la alta tasa general de éxito en los balones Mercury de 1,5mm (86\%) y Maverick de 1,5 mm. (77\%). En el caso de OTC complejas y de alta complejidad el balón de cruce más efectivo en nuestro grupo es el Maverick de 1,5 mm (100\%).

\section{Discusión:}

Es de gran utilidad llevar un registro de la efectividad de balones y guías en el tratamiento de OTC, lo que permite al intervencionista una adecuada elección del material con el consecuente ahorro de tiempo y mayor efectividad del procedimiento.

El registro que presentamos corresponde a los resultados de un período de dos años en un solo Centro en el tratamiento de OTC, en que la experiencia, agresividad y eficiencia de estas técnicas ha ido en aumento y en que indudablemente existe un sesgo de selección de material, de acuerdo a las preferencias de los operadores.

Es importante realizar una adecuada evaluación de las OTC en cuanto a las características angiográficas, lo que posibilita especificar el material más efectivo para enfrentar estas lesiones. Se han publicado estudios que muestran factores predictivos de éxito, como el largo de la lesión, presencia de bridging o experiencia del operador ${ }^{4}$, pero no se han aunado estos criterios en una clasificación para score predictivo. En este sentido el score elaborado en nuestro Laboratorio nos ha resultado de gran utilidad para predecir el éxito del procedimiento, realizar una adecuada programación del tiempo y elegir adecuadamente guías y balones que se van a utilizar.

Así en las oclusiones "simples" las guías de menos de 2 gramos de empuje en la punta (galeo Floppy) o intermedia hiodrofílica (PT2) resultan de efectividad aceptable. Rees et al., ha demostrado la utilidad de las

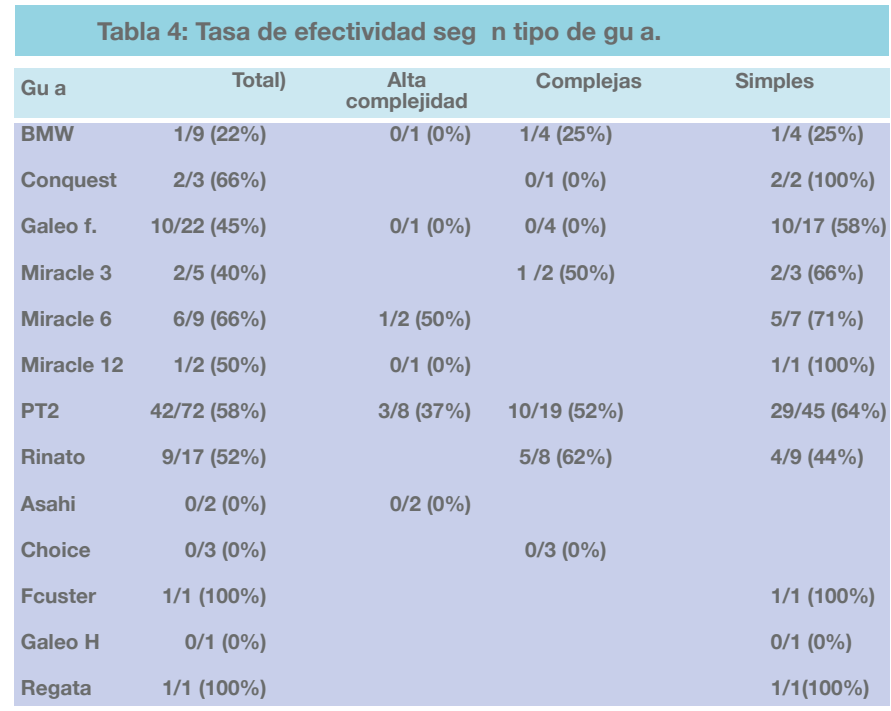




\begin{tabular}{|lllll|}
\hline \multicolumn{5}{|c}{ Tabla 5: Tasa de efectividad seg $n$ tipo de bal $n}$. \\
\hline Bal n & Total & Alta complejidad Complejas & Simples \\
\hline Maveric 1,5 & $21 / 27(77 \%)$ & $3 / 3(100 \%)$ & $5 / 5(100 \%)$ & $13 / 19(68 \%)$ \\
Maveric 2 & $14 / 20(70 \%)$ & & $5 / 5(100 \%)$ & $9 / 15(60 \%)$ \\
Mercury 1,5 & $13 / 15(86 \%)$ & $2 / 4(50 \%)$ & $3 / 3(100 \%)$ & $8 / 8(100 \%)$ \\
Mercury 2 & $3 / 5(60 \%)$ & $0 / 1(0 \%)$ & & $(75 \%)$ \\
Voyayer 1,5 & $12 / 17(70 \%)$ & $1 / 1(100 \%)$ & $7 / 8(87 \%)$ & $4 / 8(50 \%)$ \\
Elect 1,25 & $0 / 2(0 \%)$ & & $0 / 2(0 \%)$ & \\
Ryus 2 & $3 / 3(100 \%)$ & $2 / 2(100 \%)$ & $1 / 1(100 \%)$ \\
Terumo 1,25 & $2 / 2(100 \%)$ & & & $2 / 2(100 \%)$ \\
Terumo 2 & $1 / 1(100 \%)$ & & & $1 / 1(100 \%)$ \\
\hline
\end{tabular}

guías Floppy en OTC de baja complejidad ${ }^{5}$. En las lesiones "complejas" las guías floppy no hidrofílicas pierden efectividad, dando paso a guías de peso intermedio hidrofílicas (PT2) o con punta redonda como la Rinato, las que alcanzan sobre el $50 \%$ de efectividad. La utilidad de las guías hidrofílicas en el tratamiento de las OTC ya ha sido demostrado por Kahler $\mathrm{J}^{6}$. La guía Miracle de 3 gramos de empuje no hidrofílica resulta ya efectiva en estas lesiones. Luego en las estenosis de alta complejidad las propiedades hidrofílicas pierden eficacia, dando paso a guías de mayor peso en la punta como la Miracle 6 y 12 y la Conquest de 9 gramos (terminada en punta aguda), las que adquieren una mayor tasa de efectividad para este grupo de lesiones. Por otro lado al evaluar los balones de cruce, los tipos Maverick de $1,5 \mathrm{~mm}$, Mercury de $1,5 \mathrm{~mm}$ y Voyaguer de 1,5 alcanzan una tasa de efectividad bastante alta para lesiones simples o complejas. En las estenosis de alta complejidad los balones Maverick de 1,5 son los más efectivos.

Este estudio tiene las limitaciones de ser sólo un registro, por lo que la evaluación de las guías y los balones no es randomizada y en este sentido puede ser sesgada por la preferencia de los operadores. Además, el número de guías o balones no es similar para cada grupo por lo que el porcentaje de éxito puede está enmascarado por un bajo número de utilizaciones, por lo que hemos tenido el cuidado de siempre señalar junto a la tasa de éxito, el número de veces que se ha usado la guía o el balón.

En conclusión, el mantener un registro de la tasa de éxito o fracaso de los materiales en la angioplastía de las OTC resulta de gran utilidad por que permite anticipar o inferir que tan útil será la elección de dicho material, lo que es de mayor importancia en Centros donde el tratamiento de las OTC está comenzando.

\section{Limitaciones del estudio.}

El carácter de registro de este estudio conlleva un sesgo natural, ya que las lesiones estudiadas y analizadas han sido seleccionadas a priori para la realización de angioplastía.

No se incluyen algunas características importantes de las lesiones, como el tiempo de oclusión, ya que si bien este dato es de gran importancia, no se logra precisar en más del $50 \%$ de los pacientes.

El Score de complejidad utilizado nos ha resultado de gran utilidad en el enfrentamiento de las oclusiones crónicas, aunque sin duda es necesaria su validación en forma prospectiva, para su empleo en la práctica clínica de otros centros. 


\section{Referencias:}

1. Stone GW, Kandzari DE, Mehran R, Colombo A, Schwartz RS, Bailey S, et al. Percutaneous recanalization of chronically occluded coronary arteries. a consensus document. Circulation. 2005; 112: 2364-2372.

2. Lefèvre T, Louvard Y, Loubeyre C, Dumas P, Piéchaud JF, Krol M, et al. A randomized study comparing two guidewire strategies for angioplasty of chronic total coronary occlusion. Am J Cardiol. 2000; 85: 1144-7.

3. Pedreros P, Lamich R, Aguirre A, Romero C, Chamorro H. Intervencionismo en oclusiones cronicas: diseño de un score de complejidad para su aproximacion terapeutica. Rev Chil
Cardiol 2009: 28: 304

4. Maiello L, Colombo A, Gianrossi R, Mutinelli MR, Bouzon R, Thomas J, et al. Coronary angioplasty of chronic occlusions: Factors predictive of procedural success. Am Heart J 1992; 124: $581-584$.

5. Rees MR, Michalis LK, Pappa EC, Loukas S, Goudevenos JA, Sideris DA. The use of soft and flexible guidewires in the treatment of chronic total coronary occlusions by activated guidewire angioplasty. Br J Radiol 1999; 72: 162-167

6. Kähler J, Köster R, Brockhoff C, Reimers J, Baldus S, Terres $\mathrm{W}$, et al. Initial experience with a hydrophilic-coated guidewire for recanalization of chronic coronary occlusions. Catheter Cardiovasc Interv 2000; 49: 45-50 